Agro-Science Journal of Tropical Agriculture, Food, Environment and Extension Volume 12 Number 1 January 2013 pp 17 - 31

ISSN IIIY-7455

\title{
EFFECTS OF COMPOSTED OIL PALM BUNCH WASTES AND CHEMICAL FERTILIZER ON GROWTH OF OIL PALM SEEDLING UNDER WATER STRESS CONDITION
}

\author{
Ovie, S., Nnaji, G. U., Oviasogie, P.O., Osayande P.E. and Irhemu, P. \\ Department of Soil Science University of Nigeria, Nsukka. \\ Corresponding Author: oviesteve2013@yahoo.com,
}

\begin{abstract}
A greenhouse study was conducted at the University of Nigeria, Nsukka to compare the effects of sole and integrated use of composted oil palm bunch waste and chemical fertilizer for improving the growth of oil palm seedlings under water stress condition. The experiment was a $3 \times 3 \times 4$ factorial in a completely randomized design with three replications, comprising 3 factors thus irrigation intervals, NPKMg compound fertilizer and composted oil palm bunch waste. Water stress was imposed by observing irrigation intervals of 7days, 14days and 21days after irrigating the soil to field capacity. NPKMg (12:12:17:2) fertilizer was applied at $0 \mathrm{~g}$, $28 \mathrm{~g}$ and $42 \mathrm{~g}$ per seedling/polybag (based on field application rates of $0 \mathrm{Kg} / \mathrm{ha}, 1400 \mathrm{Kg} / \mathrm{ha}$ and $2100 \mathrm{Kg} / \mathrm{ha}$ ), while the composted bunch waste was applied at $0 \mathrm{~g}, 100 \mathrm{~g}, 200 \mathrm{~g}$ and $300 \mathrm{~g}$ per seedling/polybag. Result showed that 14 days irrigation intervals gave the highest $(P<0.05)$ effect on oil palm seedlings growth parameters compared to the 7 and 21 days intervals of irrigation. Similarly, application of $42 \mathrm{~g}$ NPKMg fertilizer showed the highest $(P>0.05)$ effects on growth parameters of oil palm seedlings relative to other rates of mineral fertilizers. Results also indicated that application 300gcompost/seedlings gave highest $(P<0.05)$ effect on all growth parameters compared to other compost application rates. Interaction between the composted bunch waste, chemical fertilizer and irrigation intervals had no significant effect on all the growth parameters studied. However, combined application of 42g NPKMg compound fertilizer and $300 \mathrm{~g}$ compost/seedlings was most effective in improving the growth of oil palm seedlings under 14 day intervals of irrigation and is therefore recommended.
\end{abstract}

\section{INTRODUCTION}

Every year the world population increases by 1.6 to 1.7 percent, and 90 million people are added to the consumers of agricultural products (Mannions, 1998) meaning that food production must increase to improve the nutritional needs of soaring population (Nabizade et al., 2011). Climate change constitutes one of the heightened global concerns today; it can impact agricultural productivity via changes in rainfall distribution, drought and temperature regime, as most planning and timing of farming operations are tied to the onset and duration of rainfall. Irrigation is one sure way of ameliorating climatic effects as it is used to supplement infrequent or irregular rainfall and drought periods (Idoga et al., 1998).

The West Africa oil palm (Elaeis guinensis Jaeq) is a perennial monocotyledon which belongs to the family Arecacena (Hartley, 1988). The fruit pulp and nut provide palm and kernel oil, respectively (Coley and Tinker, 2009). The oil palm industry constitutes a significant sector of the Nigerian economy providing food and raw materials for the confectionery, and oleo-chemical industry. As a result of the intensive nature of its cultivation, oil palm provides gainful employment for many Nigerians. At present, palm oil production is second to that of Soybean oil in terms of world vegetable oil production and the demand for palm oil is expected to increase in future (Yusuf, 2007; Coley, 2009).To ensure self sufficiency in palm produce concerted efforts are needed to develop strategies for improving the crop resistance to drought. The oil palm requires abundant rain above $2000 \mathrm{~mm}$ per annum well distributed throughout the year (Hartley, 1988). In Nigeria, four to five months of dry season usually occur between November and April exposing the crop to drought stress caused due to high evaporative demand, low inherent soil organic matter, and the poor moisture retention capacity that characterize the sandy nature of soils supporting oil palm in Nigeria (Babalola and Obi, 1981). The extent of the dry period and the degree of water stress are the main yield limiting factors in oil palm (Turner, 1976). In young and mature palms, moisture stress has been shown to lead to complete stomata closure 
at midday, increased inflorescence abortion, delayed growth and development and reduced yield (Turner, 1976). Isenmilla (1991) reported that irrigation interval of 14 days and above, significantly reduced vegetative growth of oil palm seedlings. Nevertheless, there is paucity of research information on the effects of integrated nutrient management on the growth of oil palm seedlings under soil water stress condition. Researchers (Aoyama et al., 1999; Olayinka, 2009) have recommended the application of organic manures to tropical soils as sources of nutrients because of the inherently low organic matter content, and low activity clays predominant in the clay mineralogy of most to spiced soils (Okusami et al., 1997). Application of farmyard manure to soil improves crop performance, soil fertility, soil organic matter, microbiological activities and soil structure for sustainable agriculture (Blair et al., 2005; Kundu et al., 2006). For healthy growth and optimal yield, nutrients must be provided to plants in correct quantity and right proportion and in a usable form at the right time. To fulfill these requirements, complementary application of chemical fertilizers and organic manures are needed. Research have indicated the beneficial effects of this practice in terms of improved soil fertility and balanced plant nutrition (Uyovbisere and Elemo, 2000; Ayoola and Agboola, 2002; Ayeni, 2008; Olayinka, 2009). The application of organic materials influences the degree of aggregation and aggregate stability and can reduce bulk density, increase porosities, water holding capacity, moisture retention and transmission, and drought resistance in plant (Cheng et al., 1988). Preliminary fertilizer trials for potted nursery experiments indicated that $28 \mathrm{~g}$ to $42 \mathrm{~g}$ NPKMg $(12: 12: 17: 2)$ applied at two or three equal doses seemed adequate for optimum oil palm seedling production (Onwubuya, 1982; Ugbah, 2008). Similarly, Aisueni et al. (2000) reported that composted oil palm bunch waste when applied at $150 \mathrm{~g}$ per polybag enhanced nursery seedlings production. The rate could be reduced to $100 \mathrm{~g}$ per oil palm seedlings when complemented with $12 \mathrm{~g}$ of NPKMg 12:12:17:2 inorganic fertilizer for better performance (Aisueni et al., 2000). The present study was designed to compare the effects of sole and integrated use of composted oil palm bunch waste and NPK fertilizer for improving the growth of oil palm seedlings under water stress condition.

\section{MATERIALS AND METHODS}

The study site: This study was conducted at the greenhouse of the Department of Soil Science, University of Nigeria Nsukka. Nsukka, is in Enugu State in the derived savannah agro- ecological zone of Nigeria. The area lies within latitude $06^{\circ} 52^{\prime} \mathrm{N}$ and longitude $07^{\circ} 24^{\prime} \mathrm{E}$; with a mean elevation of 419 metres above sea level. Humid tropical climate prevails with annual rainfall of $1700-1800 \mathrm{~mm}$. The rainfall pattern is bimodal and falls between April and October, while the dry season is between November and March. The area is characterized with mean annual maximum (day) and minimum (night) temperatures of $31^{\circ} \mathrm{C}$ and $21^{\circ} \mathrm{C}$, respectively, while the average relative humidity is rarely below 60\% (Asadu, 2002). The soil used for the study has been classified as Typic Kandiustult, derived from a false bedded sand stone (Akamigbo and Igwe, 1990).

\section{Treatment Application}

Soil sampling and analysis: Representative topsoil from $0-15 \mathrm{~cm}$ was collected and sieved using $2 \mathrm{~mm}$ mesh. Some portions were analyzed for initial physico-chemical properties.

Preparation of composted oil palm bunch waste (COBW): Materials used for the composting were oil palm bunch waste, poultry droppings and palm oil mill effluent. Oil palm bunch wastes were shredded into smaller particles. The shredded bits were packed in windrows (aerated compartment) and mixed with poultry droppings in a ratio of $4: 1$ (bunch waste: poultry droppings). Palm oil effluent was added to increase microbial activity and to reduce the $\mathrm{C} / \mathrm{N}$ ratio. The heap was turned weekly and moistened with the effluent for proper degradation. The composting process lasted for 10 weeks after which the compost was air dried in preparedness for chemical analysis. The overall procedure was the method adopted by Hang, (1993) and Catton (1983)

Chemical analysis of the uncomposted and composted materials used for the study: Samples of the base materials including the oil palm bunch waste, palm oil mill effluent, poultry manure and the final compost were crushed and passed through a $2 \mathrm{~mm}$ sieve and chemical properties such as $\mathrm{pH}$, total $\mathrm{N}$, available $\mathrm{P}$, exchangeable bases and organic carbon were determined.

Pre-nursery establishment of oil palm seedlings: Pre nursery polybag measuring $12.5 \mathrm{x}$ $25 \mathrm{~cm}$ were filled with sieved topsoil and presprouted oil palm seeds sourced from the Nigerian Institute for Oil Palm Research (NIFOR), Benin City were planted out. A shed was erected for the pre-nursery to prevent the seeds from desiccation, and cultural practices such as weeding, watering and disease control were observed. 
Ovie, S., Nnaji, G. U., Oviasogie, P.O., Osayande P.E. and Irhemu, P.

Main-nursery establishment: At three leaf stage, i.e. after 10 weeks of growth at the prenursery, all healthy seedlings of uniform growth were selected and transplanted singly into each prepared main nursery poly bag measuring $40 \mathrm{~cm} \times 45 \mathrm{~cm}$. The main nursery polybags contained a mixture of $7 \mathrm{~kg}$ sieved topsoil and the composted oil palm waste according to the treatment levels. They were carefully arranged in the greenhouse and watered uniformly for a period of three weeks in order to obtain good plant establishment. The various irrigation intervals were then established every 7, 14 and 21 days. Cultural practices such as weeding and pest control were observed. The NPKMg 12:12:17:2 compound fertilizer was applied using the ring method according to the treatment levels (Onwubuya, 1982). The oil weeding was done regularly by hand picking and Dithane M45 was sprayed every fortnight to control moderate infection of freckle on the seedings.

Experimental Design: The experiment was laid out as $3 \times 3 \times 4$ factorial in a completely randomized design with three replications. The factors included NKPMg compound fertilizer, composted oil palm bunch waste and irrigation intervals. NPKMg (12:12:17:2) fertilizer was applied at three levels $0 \mathrm{~g}, 28 \mathrm{~g}$ and $42 \mathrm{~g}$ per seedling/polybag (based on field application rates of $0 \mathrm{Kg} / \mathrm{ha}, 1400 \mathrm{Kg} / \mathrm{ha}$ and $2100 \mathrm{Kg} / \mathrm{ha}$ ). The $28 \mathrm{~g}$ dose was applied in two split doses of $14 \mathrm{~g}$ at 3 and 5 months after planting, while $42 \mathrm{~g}$ dose was applied in three equal doses of $14 \mathrm{~g}$ at 3,5 and 8 months after planting. Water stress was imposed by observing irrigation intervals of 7days, 14days and 21days after irrigating the soil to field capacity. The composted empty fruit bunch wastes were applied at four levels, thus, $0 \mathrm{~g}, 100 \mathrm{~g}, 200 \mathrm{~g}$ and $300 \mathrm{~g}$ per seedling/polybag.

Irrigation procedure: After the three weeks period of uniform watering in the greenhouse, the polybags were weighed $48 \mathrm{hrs}$ later so as to determine the weight at field capacity by assuming that free gravitational water would have stopped draining (Richard and Weaver, 1944). The water stress was imposed by observing irrigation intervals at every 7,14 and 21 days from the field capacity water content. The nursery polybags were also weighed at the different irrigation intervals so as to determine the level of soil water depletion. The deficit in soil water at the different irrigation intervals was ascribed to be the approximate water loss by evapotranspiration. The deficits were estimated and the calculated amount of irrigation water (volume) was added to raise the soil water to the predetermined field capacity following the procedures of Boutraa and Sanders (2001).

Growth measurement: Plant height which was measured as the distance from the soil surface to the tallest leaf and the number of leaves per seedlings was determined by counting the fully unfurled leaves on each plant. Stem girth was measured with a screw gauge placed at the maximum girth of the seedlings. The leaf area was estimated as the leaf length multiplied by its maximum width multiplied by 0.05 following, Harden et al. (1965). Data were collected at 5, 8 and 12 months after planting, i.e., 3, 6 and 10 months after treatment application.

\section{Laboratory methods}

Particle size distribution was determined by Bouyoucos (1962) hydrometer method. Soil pH was determined in 1:2.5 soil to water ratio using $\mathrm{pH}$ meter (Mclean, 1965). Total nitrogen was determined by semi-micro kjeldahl method (Bremner, 1996) using selenium tables as catalyst. Organic carbon was determined by chromic acid wet oxidation method of Nelson and Sommers (1982), while organic matter was determined by multiplying percentage organic carbon by 1.724. Available phosphorus was determined using Bray No 1 method (Bray and Kurtz, 1945). Exchangeable bases were determined by $1 \mathrm{~N}$ neutral $\mathrm{NH}_{4} \mathrm{OAC}$ saturation method of Grant (1982).

Data Analysis: The statistical analysis was performed using Genstat Statistical Package for the analysis of variance (ANOVA).Treatment means were compared using the Fisher's Least Significant Difference (F-LSD) at 5\% probability.

\section{RESULTS}

Table 1 showed the initial properties of the soil before treatment application. The soil was sandy loam with a high percentage of sand and low percentages of clay and silt. The soil was low in $\mathrm{pH}$, total $\mathrm{N}$, available $\mathrm{P}$, exchangeable $\mathrm{K}, \mathrm{Ca}$, $\mathrm{Mg}$, Na and organic matter, respectively. Table 2 showed the chemical properties of the materials used during the nursery media composting and composted oil palm bunch waste. Chemical analysis revealed higher levels of total nitrogen; exchangeable Magnesium, exchangeable Calcium, and lowest $\mathrm{C} / \mathrm{N}$ ratio values of poultry manure compared to palm oil mill effluent (POME) and shredded oil palm bunch waste. Uncomposted oil palm bunch waste had the highest $\mathrm{pH}$ value, exchangeable $\mathrm{K}$, organic carbon and $\mathrm{C} / \mathrm{N}$ ratio relative to poultry manure and palm oil mill effluent respectively (Table 2). Chemical analysis of the composted oil palm 
bunch waste showed higher nutrient composition in terms of total nitrogen, available phosphorus, exchangeable bases and $\mathrm{pH}$ relative to the raw materials (Table 2).The composted oil palm bunch waste had a lower $\mathrm{C} / \mathrm{N}$ ratio compared to the raw oil palm bunch waste used for the composting.

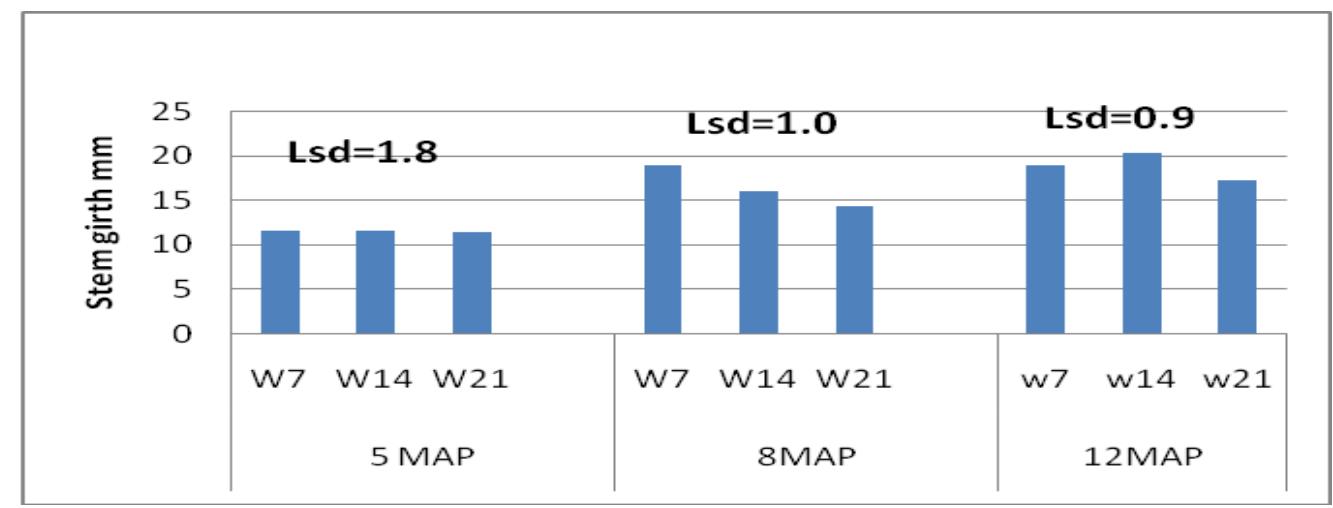

Fig 1: Main effect of irrigation intervals on stem girth at 5, 8 and 12MAP

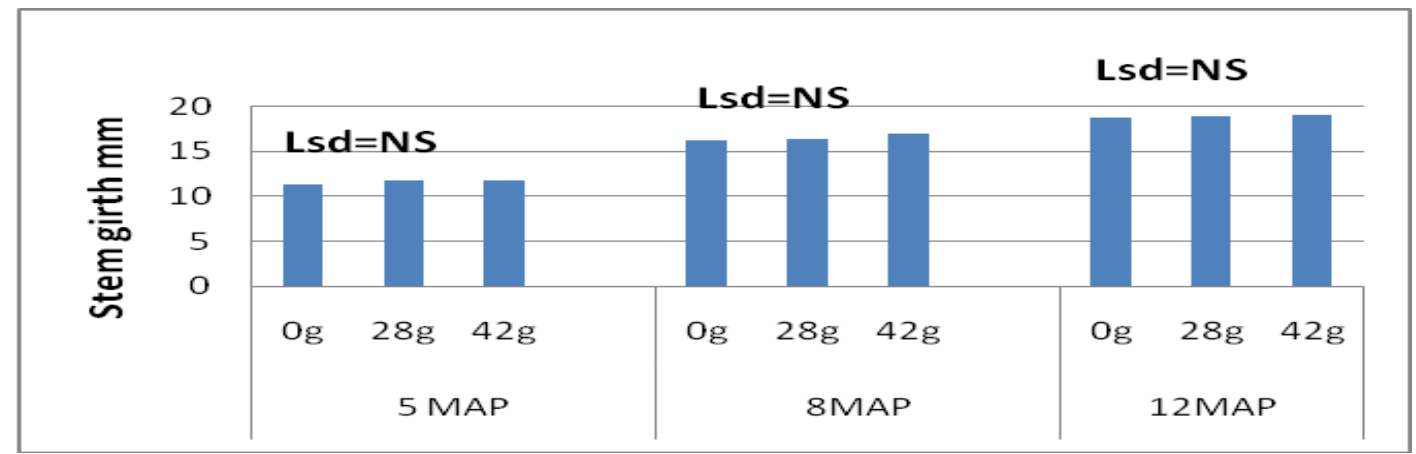

Fig 2: Main effect of NPK fertilizer on stem girth at 5, 8 and 12MAP

Table 1: Initial physico-chemical properties of the soil used for the study

\begin{tabular}{ll}
\hline Characteristics & Values \\
\hline Fine Sand $(\%)$ & 26 \\
Coarse Sand $(\%)$ & 50 \\
Silt $(\%)$ & 11 \\
Clay $(\%)$ & 13 \\
Textural class & Sandy Loam \\
$\mathrm{pH}\left(\mathrm{H}_{2} \mathrm{O}\right)$ & 4.4 \\
Total Nitrogen $(\%)$ & 0.03 \\
Organic Matter $(\%)$ & 1.15 \\
Organic Carbon $(\%)$ & 0.67 \\
Available Phosphorus. $(\mathrm{mg} / \mathrm{kg})$ & 9.14 \\
Exchangeable Potassium $(\mathrm{cmol} / \mathrm{kg})$ & 0.06 \\
Exchangeable Calcium $(\mathrm{cmol} / \mathrm{kg})$ & 2.02 \\
Exchangeable Sodium $(\mathrm{cmol} / \mathrm{kg})$ & 0.37 \\
\hline
\end{tabular}


Ovie, S., Nnaji, G. U., Oviasogie, P.O., Osayande P.E. and Irhemu, P.

Table 2: Chemical properties of the materials used for composting oil palm bunch waste and composted oil palm bunch waste

\begin{tabular}{lllll}
\hline Properties & Poultry Manure & $\begin{array}{l}\text { Palm oil } \\
\text { mill effluent }\end{array}$ & $\begin{array}{l}\text { Shredded oil } \\
\text { oil palm bunch } \\
\text { waste }\end{array}$ & $\begin{array}{c}\text { Composted oil palm } \\
\text { bunch } \\
\text { waste }\end{array}$ \\
\hline $\mathrm{pH}\left(\mathrm{H}_{2} \mathrm{0}\right)$ & & & 7.3 & 8.2 \\
$\mathrm{EC}(\mathrm{ds} / \mathrm{m})$ & 5.8 & 3.3 & 10.44 & 5.4 \\
Nitrogen $(\%)$ & 18.80 & 7.44 & 0.36 & 2.24 \\
Potassium $(\%)$ & 1.62 & 0.44 & 1.30 & 9.81 \\
Magnesium $(\%)$ & 1.05 & 0.49 & 0.24 & 3.12 \\
Calcium $(\%)$ & 0.32 & 0.07 & 0.49 & 4.82 \\
Phosphorus $(\%)$ & 1.89 & 0.42 & 0.04 & 0.65 \\
C/N ratio & 0.05 & 0.02 & 35.47 & 17.85 \\
Carbon $(\%)$ & 0.39 & 7.00 & 12.77 & 40.00 \\
\hline
\end{tabular}

Effects of treatments on growth of oil palm seedlings

Stem girth: Figure 1 showed the main effects of irrigation intervals on stem girth of oil palm seedlings at 5, 8 and 12 months after planting (MAP). At 5MAP the highest $(\mathrm{P}<0.05)$ value was obtained at irrigation intervals of 14 days. On the contrary, at 8MAP, highest $(\mathrm{P}<0.05)$ was obtained under 7 days irrigation intervals. At 12MAP, the largest girth was observed at 14 days irrigation intervals. Figure 2 showed the main effects of chemical fertilizer on stem girth of oil palm seedlings at 5,8 and 12 MAP. At 5MAP the highest $(\mathrm{P}>0.05)$ value was obtained at $28 \mathrm{gNPK}$. At $8 \mathrm{MAP}$, the highest value was observed at $42 \mathrm{gNPK}$ but showed no statistical difference. Result obtained at 12MAP, had the highest $(\mathrm{P}>0.05)$ value at $42 \mathrm{gNPK}$. Figure 3 showed main effect of composted oil palm bunch waste on stem girth of oil palm seedlings at 5, 8 and 12 MAP. At 5MAP, highest $(\mathrm{P}<0.05)$ value was obtained at $300 \mathrm{gEFB}$. Similar trend were observed at 8 and 12MAP.

Table 3 showed effects of compost and chemical fertilizer on stem girth of oil palm seedlings under different irrigation intervals at 5, 8 and 12 months after planting. At 5MAP, highest stem girth value was observed at 14 days intervals of irrigation. At $8 \mathrm{MAP}$, every 7 day irrigation intervals recorded the highest and at 12MAP, 14 day intervals of irrigation gave the highest stem girth compared to 7 and 21 days irrigation intervals respectively. However at 5MAP, fertilizing oil palm seeding with $42 \mathrm{gNPKMg}+300 \mathrm{gcompost}$ and $28 \mathrm{gNPKMg}+200 \mathrm{~g}$ Compost gave the highest stem girth values followed by those fertilized with $\quad 0 \mathrm{gNPKMg}+200 \mathrm{gcompost}$ and $42 \mathrm{gNPKMg}+200 \mathrm{gCompost}$. Lowest values were obtained from the control plants $(0 \mathrm{gNPKMg}+0 \mathrm{~g}$ compost), likewise the plants fertilized with $28 \mathrm{gNPKMg}+0 \mathrm{gcompost}$ and $42 \mathrm{gNPKMg}+0 \mathrm{gcompost}$. The interaction effect between compost, irrigation intervals and chemical fertilizer were not statistically significant $(P>0.05)$ throughout the growth period. At 8MAP, fertilizing with $42 \mathrm{gNPKMg}+300 \mathrm{~g}$ compost and $0 \mathrm{gNPKMg}+300$ gcompost gave the highest values followed by those fertilized with $28 \mathrm{gNPKMg}+200 \mathrm{gcompost}$ and 0gNPKMg+100gcompost. Lowest values were obtained from the control plants (0gNPKMg+0gcompost) likewise the plants fertilized with $28 \mathrm{gNPKMg}+0 \mathrm{gcompost}$ and $42 \mathrm{gNPKMg}+0 \mathrm{gcompost}$. The interaction effect between compost, irrigation intervals and chemical fertilizer was not significant $(\mathrm{P}>0.05)$ at 8MAP (Table 3). At 12MAP, fertilizing oil palm seedling with $42 \mathrm{gNPKMg}+300 \mathrm{gcompost}$ and $0 \mathrm{~g} \mathrm{NPKMg}+300 \mathrm{gcompost}$ gave the highest values followed by those fertilized with $28 \mathrm{gNPKMg}+200 \mathrm{gcompost}$ and $28 \mathrm{gNPKMg}+300 \mathrm{gcompost}$. Lowest values were obtained from the control plant (0gNPKMg+0gcompost) likewise the plants fertilized with $28 \mathrm{gNPKMg}+0 \mathrm{gcompost}$ and $42 \mathrm{gNPKMg}+0 \mathrm{gcompost}$. The interaction effect between compost, irrigation intervals and chemical fertilizer was not significant $(\mathrm{P}>0.05)$ at 12 MAP (Table 3).

Plant Height: Figure 4 showed main effects of irrigation intervals on plant height of oil palm seedlings at 5, 8 and $12 \mathrm{MAP}$. At 5MAP the highest $(\mathrm{P}<0.05)$ value was obtained at irrigation intervals of 14 days. At 8MAP, highest significant value was observed under 7days irrigation intervals. At 12MAP, highest $(\mathrm{P}<0.05)$ value was recorded at 14 days irrigation intervals. Figure 5 showed the main effects of chemical fertilizer on plant height of oil palm seedlings at 5,8 and 12 MAP. At 5MAP the highest $(\mathrm{P}<0.05)$ value was obtained at $28 \mathrm{gNPK}$. At both 8 and $12 \mathrm{MAP}$, the highest $(\mathrm{P}<0.05)$ values were observed at $42 \mathrm{gNPK}$. Figure 6 showed main effects of composted EFB on plant height of oil palm seedlings at 5, 8 and 12 MAP. At 5MAP, the highest $(\mathrm{P}<0.05)$ value was obtained at $300 \mathrm{gEFB}$ relative to the control 
(0gEFB). Similar trend were observed at 8 and 12 MAP (Figure 6)

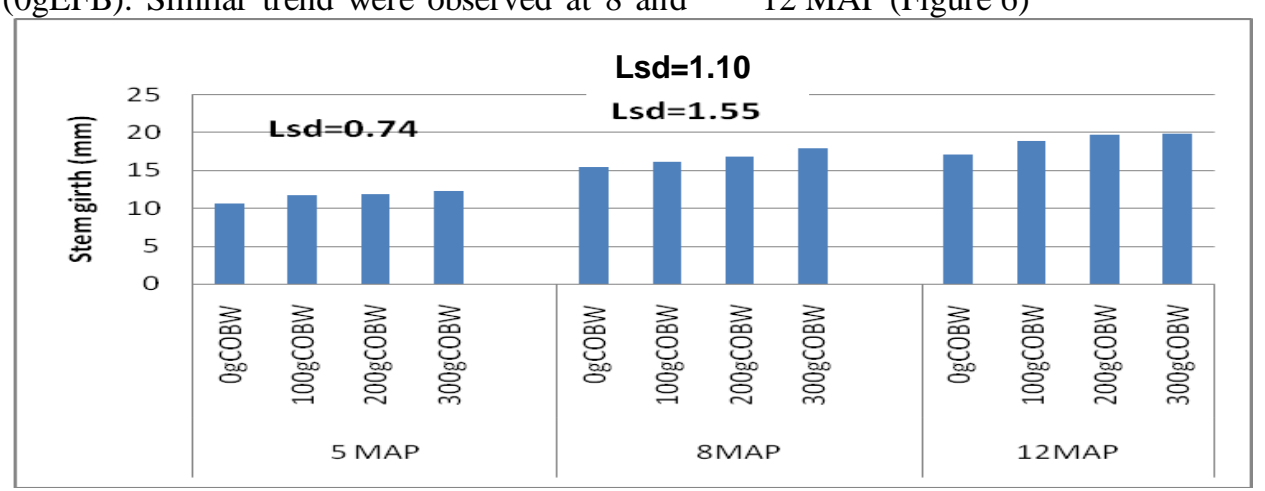

Fig 3: Main effect of composted oil palm bunch waste on stem girth at 5, 8 and 12MAP

Table 3: Effects of compost and chemical fertilizer on stem girth $(\mathrm{mm})$ of oil palm seedlings under different irrigation intervals at 5,8 and 12 months after planting (i.e. 3 , 6 and 10 months after treatment application)

\begin{tabular}{|c|c|c|c|c|c|c|c|c|c|c|c|c|}
\hline \multirow[b]{2}{*}{ Nutrient combinations } & \multicolumn{4}{|c|}{$\begin{array}{c}5 \mathrm{MAP} \\
\text { Irrigation intervals (days) }\end{array}$} & \multicolumn{4}{|c|}{$\begin{array}{l}8 \text { MAP } \\
\text { Irrigation intervals (days) }\end{array}$} & \multicolumn{4}{|c|}{$\begin{array}{c}12 \text { MAP } \\
\text { Irrigation intervals (days) }\end{array}$} \\
\hline & 7 & 14 & 21 & Mean & 7 & 14 & 21 & Mean & 7 & 14 & 21 & Mean \\
\hline $\mathrm{T} 1=0 \mathrm{gNPK}+0 \mathrm{gCOBW}$ & 10.02 & 11.68 & 11.08 & 10.92 & 17.27 & 14.69 & 12.15 & 14.70 & 16.03 & 19.03 & 15.54 & 16.86 \\
\hline $\mathrm{T} 2=0 \mathrm{gNPK}+100 \mathrm{gCOBW}$ & 12.22 & 11.33 & 12.37 & 11.97 & 19.24 & 16.38 & 14.67 & 16.76 & 18.83 & 20.70 & 18.30 & 19.27 \\
\hline $\mathrm{T} 3=0 \mathrm{gNPK}+200 \mathrm{gCOBW}$ & 12.22 & 12.26 & 12.10 & 12.19 & 20.06 & 15.72 & 13.57 & 16.45 & 18.30 & 21.20 & 17.12 & 18.87 \\
\hline $\mathrm{T} 4=0 \mathrm{gNPK}+300 \mathrm{gCOBW}$ & 12.62 & 11.82 & 11.01 & 11.82 & 20.62 & 17.00 & 15.13 & 17.58 & 19.47 & 22.53 & 18.75 & 20.25 \\
\hline $\mathrm{T} 5=28 \mathrm{gNPK}+0 \mathrm{gCOBW}$ & 9.62 & 11.02 & 10.23 & 10.29 & 18.69 & 14.26 & 13.45 & 15.46 & 17.83 & 17.94 & 14.58 & 16.78 \\
\hline $\mathrm{T} 6=28 \mathrm{gNPK}+100 \mathrm{gCOBW}$ & 10.92 & 11.01 & 10.90 & 10.94 & 18.72 & 15.52 & 15.16 & 16.46 & 20.77 & 20.35 & 17.05 & 19.39 \\
\hline $\mathrm{T} 7=28 \mathrm{gNPK}+200 \mathrm{~g} \mathrm{COBW}$ & 12.89 & 11.36 & 12.62 & 12.29 & 19.59 & 16.76 & 15.35 & 17.23 & 20.10 & 20.72 & 18.00 & 19.60 \\
\hline $\mathrm{T} 8=28 \mathrm{gNPK}+300 \mathrm{~g} \mathrm{COBW}$ & 11.80 & 11.41 & 10.90 & 11.37 & 16.63 & 17.61 & 14.66 & 16.30 & 20.92 & 20.27 & 17.32 & 19.50 \\
\hline $\mathrm{T} 9=42 \mathrm{gNPK}+0 \mathrm{~g} \mathrm{COBW}$ & 11.80 & 11.41 & 10.90 & 11.37 & 19.26 & 16.98 & 11.45 & 15.58 & 16.68 & 18.53 & 16.92 & 17.37 \\
\hline $\mathrm{T} 10=42 \mathrm{gNPK}+100 \mathrm{gCOBW}$ & 12.22 & 11.74 & 12.19 & 12.05 & 18.87 & 15.08 & 14.35 & 16.10 & 18.54 & 19.50 & 16.13 & 18.05 \\
\hline $\mathrm{T} 11=42 \mathrm{gNPK}+200 \mathrm{gCOBW}$ & 11.65 & 12.28 & 12.79 & 12.36 & 17.26 & 16.24 & 15.90 & 16.46 & 19.57 & 19.82 & 19.02 & 19.47 \\
\hline $\mathrm{T} 12=42 \mathrm{gNPK}+300 \mathrm{gCOBW}$ & 11.31 & 12.98 & 12.79 & 12.36 & 21.23 & 16.25 & 16.14 & 17.87 & 20.17 & 22.53 & 18.49 & 20.39 \\
\hline Mean & 11.57 & 11.63 & 11.47 & & 18.96 & 16.04 & 14.33 & & 18.93 & 20.26 & 17.27 & \\
\hline F-LSD $0.05 \mathrm{~A}$ & & 1.80 & & & & 1.00 & & & & 0.90 & & \\
\hline F-LSD $0.05 \mathrm{~B}$ & $\mathrm{~N}$ & & & & & NS & & & & NS & & \\
\hline F-LSD $0.05 \mathrm{C}$ & 0 . & & & & & 1.55 & & & & 1.10 & & \\
\hline F-LSD $0.05 \mathrm{~A} \times \mathrm{B} \times \mathrm{C}$ & $\mathrm{N}$ & & & & & NS & & & & NS & & \\
\hline
\end{tabular}

$\mathrm{A}=$ irrigation interval, $\mathrm{B}=$ chemical fertilizer $\mathrm{C}=$ composted oil palm bunch waste. $\mathrm{A} \times \mathrm{B} \times \mathrm{C}=$ interactions, $\mathrm{COBW}=$ composted oil palm bunch waste

Table 4 showed the effects of compost and chemical fertilizer on plant height of oil palm seedlings under different irrigation intervals at 5 , 8 and 12 months after planting. At 5MAP, highest plant height value was observed at 14 day irrigation intervals. At 8 MAP, 7 day irrigation intervals recorded the highest value, while at 12MAP 14 day irrigation intervals gave the highest plant height value compared to 7 and 21 days irrigation intervals respectively. At 5MAP, fertilizing oil palm seedling with $0 \mathrm{gNPKMg}+300$ gcompost

and $28 \mathrm{gNPKMg}+300 \mathrm{gcompost}$ gave the highest values followed by those fertilized with $0 \mathrm{gNPKMg}+100 \mathrm{gcompost}$ and $28 \mathrm{gNPKMg}+200 \mathrm{gCompost}$. Lowest values were obtained from the control plant (0gNPKMg+0gcompost) likewise the plants fertilized with $28 \mathrm{gNPKMg}+0 \mathrm{gcompost}$ and $42 \mathrm{gNPKMg}+0 \mathrm{gcompost}$. The interaction effect between compost, irrigation intervals and chemical fertilizer were not statistically significant $(\mathrm{P}>0.05)$.

At 8MAP, soil amended with $28 \mathrm{gNPKMg}+300 \mathrm{gcompost}$ and $42 \mathrm{gNPKMg}+300$ gcompost gave the highest values followed by those fertilized with $28 \mathrm{~g}$ $\mathrm{NPKMg}+200 \mathrm{~g}$ compost and $28 \mathrm{gNPKMg}+200 \mathrm{gcompost}$. Lowest values were obtained from the control plants (0gNPKMg+0gcompost), likewise the plants fertilized with $28 \mathrm{gNPKMg}+0 \mathrm{gcompost}$ and $42 \mathrm{gNPKMg}+0 \mathrm{gcompos}$. The interaction effect between compost, irrigation intervals and chemical fertilizer was not significant $(\mathrm{P}>0.05)$.

At 12 MAP, fertilizing oil palm seedling with $42 \mathrm{gNPKMg}+300 \mathrm{gcompost}$ and $28 \mathrm{gNPKMg}+200 \mathrm{gcompost}$ gave the highest values followed by $0 \mathrm{gNPKMg}+300 \mathrm{~g}$ compost $42 \mathrm{gNPKMg}+200 \mathrm{gcompost}$. Lowest values were obtained from the control plants (0gNPKMg+0gcompost), likewise the plants fertilized with $28 \mathrm{gNPKMg}+0 \mathrm{gcompost}$ and 
$42 \mathrm{gNPKMg}+0 \mathrm{gcompost}$. The interaction effect between compost, irrigation intervals and chemical fertilizer was not significant $(\mathrm{P}>0.05)$.

Leaf area: Figure 7 showed main effects of irrigation intervals on leaf area of oil palm seedlings at 5, 8 and $12 \mathrm{MAP}$. At 5MAP, the highest $(\mathrm{P}>0.05)$ value was obtained at irrigation intervals of 14 days. At 8MAP, the highest $(\mathrm{P}<0.05)$ value was observed under 7days irrigation intervals. At 12MAP, 14 days irrigation intervals had the highest value (Fig. 7). Figure 8 showed that the main effects of chemical fertilizer were not statistically significant on leaf area of oil palm seedlings throughout the growth period. Figure 9 showed the main effects of composted oil palm bunch waste on leaf area of oil palm seedlings at 5,8 and 12 MAP. At 5MAP, the highest $(\mathrm{P}<0.05)$ value was obtained at $300 \mathrm{gcomposted}$ oil palm bunch waste relative to the control. Similar trend were observed at 8 and 12 MAP.

Table 5 showed the effects of compost and chemical fertilizer on leaf area of oil palm seedlings under different irrigation intervals at 5, 8 and 12 months after planting. At 5MAP, highest leaf area value was observed at 14 day irrigation intervals. At 8 MAP, 7day irrigation intervals recorded the highest value, while at 12MAP, 14 day irrigation intervals gave the highest leaf area compared to 7 and 21 days irrigation intervals respectively. However, at 5MAP, fertilizing oil palm seeding with $0 \mathrm{gNPKMg}+100 \mathrm{gcompost}$ and $42 \mathrm{gNPKMg}+300 \mathrm{gcompost}$ gave the highest values followed by those fertilized with $0 \mathrm{gNPKMg}+300$ gcompost and $28 \mathrm{gNPKMg}+300 \mathrm{gCompost}$. Lowest values were obtained from the control plants (0gNPKMg+0gcompost), likewise the plants fertilized with $28 \mathrm{gNPKMg}+0 \mathrm{gcompost}$ and $42 \mathrm{gNPKMg}+0 \mathrm{gcompost}$. The interaction effect between compost, irrigation intervals and chemical fertilizer were not statistically significant $(\mathrm{P}>0.05)$ (Table 5)

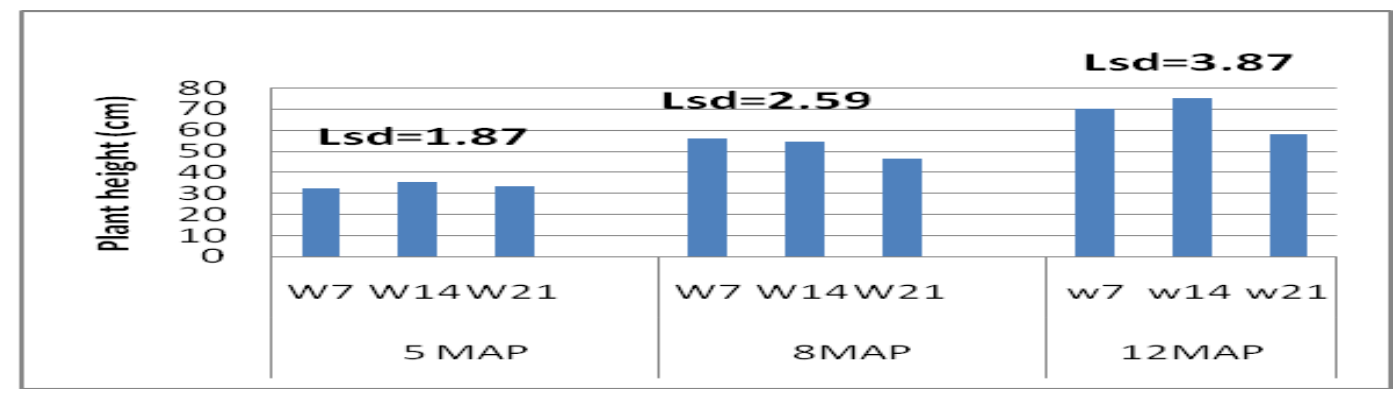

Fig 4: Main effects of irrigation intervals on plant height $(\mathrm{cm})$ at 5, 8 and 12 MAP

Table 4: Effects of compost and chemical fertilizer on plant height $(\mathrm{cm})$ of oil palm seedlings under different irrigation intervals at 5,8 and 12 months after planting (i.e. 3, 6 and 10 months after treatment application)

\begin{tabular}{|c|c|c|c|c|c|c|c|c|c|c|c|c|}
\hline \multirow[b]{2}{*}{ Nutrient combinations } & \multicolumn{3}{|c|}{$\begin{array}{c}5 \text { MAP } \\
\text { Irrigation intervals (days) }\end{array}$} & \multirow[b]{2}{*}{ Mean } & \multicolumn{3}{|c|}{$\begin{array}{c}8 \text { MAP } \\
\text { Irrigation intervals (days) }\end{array}$} & \multicolumn{5}{|c|}{$\begin{array}{c}12 \text { MAP } \\
\text { Irrigation intervals (days) }\end{array}$} \\
\hline & 7 & 14 & 21 & & 7 & 14 & 21 & Mean & 7 & 14 & 21 & Mean \\
\hline $\mathrm{T} 1=0 \mathrm{gNPKMg}+0 \mathrm{gCOBW}$ & 27.03 & 31.15 & 31.27 & 29.81 & 51.27 & 46.93 & 40.67 & 46.29 & 52.00 & 69.00 & 56.67 & 59.22 \\
\hline $\mathrm{T} 2=0 \mathrm{gNPK}+100 \mathrm{gCOBW}$ & 37.55 & 36.85 & 34.68 & 36.36 & 52.00 & 53.33 & 50.07 & 51.80 & 62.33 & 66.67 & 62.33 & 63.77 \\
\hline $\mathrm{T} 3=0 \mathrm{gNPK}+200 \mathrm{gCOBW}$ & 32.58 & 36.07 & 32.85 & 33.83 & 57.60 & 51.13 & 46.33 & 51.68 & 76.67 & 76.00 & 53.33 & 68.66 \\
\hline $\mathrm{T} 4=0 \mathrm{gNPK}+300 \mathrm{gCOBW}$ & 32.05 & 42.45 & 37.75 & 37.41 & 50.67 & 56.67 & 48.57 & 51.97 & 82.00 & 81.67 & 65.33 & 76.33 \\
\hline $\mathrm{T} 5=28 \mathrm{gNPK}+0 \mathrm{gCOBW}$ & 30.80 & 33.50 & 29.43 & 31.24 & 51.27 & 51.53 & 42.00 & 48.26 & 61.00 & 65.33 & 49.50 & 58.61 \\
\hline $\mathrm{T} 6=28 \mathrm{gNPK}+100 \mathrm{gCOBW}$ & 29.18 & 32.55 & 33.10 & 31.61 & 55.77 & 56.33 & 49.67 & 53.92 & 66.00 & 65.00 & 56.57 & 62.52 \\
\hline $\mathrm{T} 7=28 \mathrm{gNPK}+200 \mathrm{gCOBW}$ & 35.73 & 35.68 & 34.80 & 35.40 & 55.23 & 56.67 & 48.57 & 55.15 & 69.33 & 68.00 & 56.67 & 64.66 \\
\hline $\mathrm{T} 8=2 \mathrm{gNPK}+300 \mathrm{gCOBW}$ & 35.50 & 36.12 & 28.87 & 36.83 & 64.67 & 61.67 & 48.57 & 58.34 & 82.07 & 84.33 & 64.67 & 77.22 \\
\hline $\mathrm{T} 9=42 \mathrm{gNPK}+0 \mathrm{gCOBW}$ & 31.50 & 32.50 & 31.00 & 31.67 & 54.03 & 48.50 & 40.03 & 47.52 & 68.33 & 72.00 & 48.33 & 68.88 \\
\hline $\mathrm{T} 10=42 \mathrm{gNPK}+100 \mathrm{gCOBW}$ & 32.58 & 35.60 & 31.25 & 33.14 & 58.33 & 53.00 & 40.93 & 50.75 & 69.33 & 79.50 & 53.33 & 67.38 \\
\hline $\mathrm{T} 11=42 \mathrm{gNPK}+200 \mathrm{gCOBW}$ & 32.23 & 36.20 & 34.33 & 34.25 & 51.63 & 58.33 & 47.37 & 52.44 & 67.67 & 82.50 & 61.67 & 70.94 \\
\hline $\mathrm{T} 12=42 \mathrm{gNPK}+300 \mathrm{gCOBW}$ & 31.50 & 36.62 & 35.80 & 34.64 & 60.53 & 60.50 & 50.00 & 57.01 & 79.67 & 87.33 & 67.00 & 78.00 \\
\hline Mean & 32.35 & 35.44 & 35.34 & & 55.67 & 54.55 & 46.08 & & 69.75 & 74.78 & 57.95 & \\
\hline F-LSD $0.05 \mathrm{~A}$ & \multicolumn{4}{|c|}{1.87} & \multicolumn{3}{|c|}{2.59} & \multicolumn{5}{|c|}{3.87} \\
\hline F-LSD $0.05 \mathrm{~B}$ & \multicolumn{3}{|c|}{1.80} & & \multicolumn{3}{|c|}{2.59} & \multicolumn{5}{|c|}{3.87} \\
\hline F-LSD $0.05 \mathrm{C}$ & \multicolumn{3}{|c|}{2.10} & & \multicolumn{3}{|c|}{3.00} & \multicolumn{5}{|c|}{4.47} \\
\hline F-LSD $0.05(\mathrm{~A} \times \mathrm{B} \times \mathrm{C})$ & \multicolumn{2}{|c|}{ NS } & & & \multicolumn{3}{|c|}{ NS } & \multicolumn{5}{|c|}{ NS } \\
\hline
\end{tabular}

$\mathrm{A}=$ irrigation interval, $\mathrm{B}=$ chemical fertilizer $\mathrm{C}=$ composted oil palm bunch waste. $\mathrm{A} \times \mathrm{B} \times \mathrm{C}=$ interactions, $\mathrm{COBW}=$ composted oil palm bunch waste 


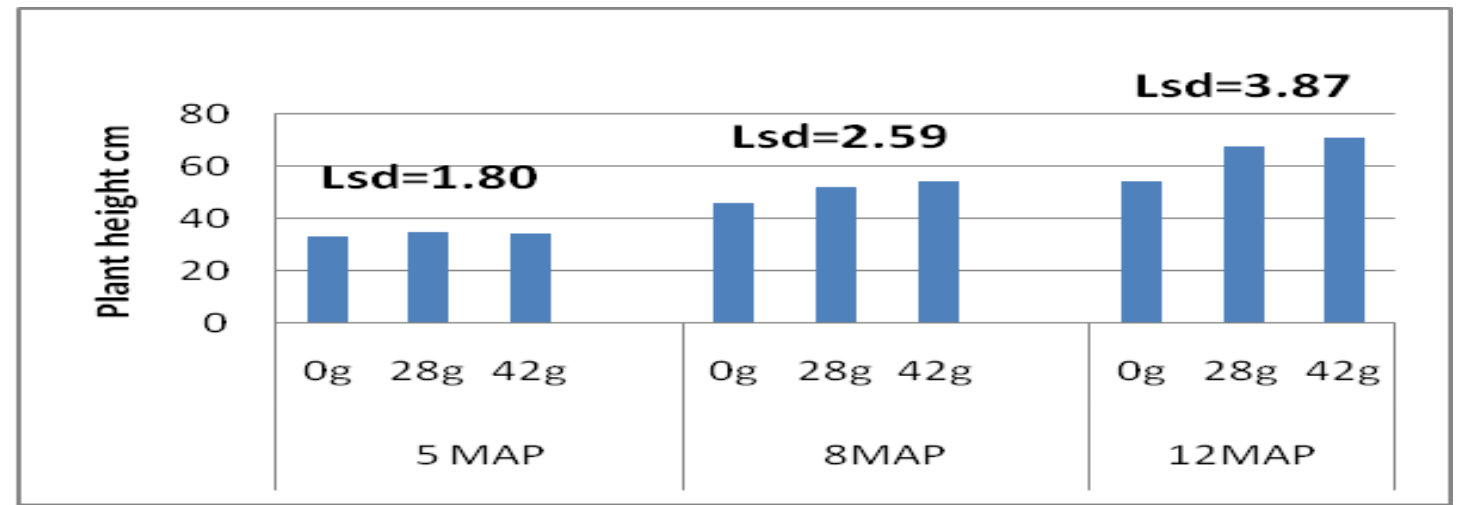

Fig 5: Main effects of chemical fertilizer on plant height at 5, 8 and $12 \mathrm{MAP}$

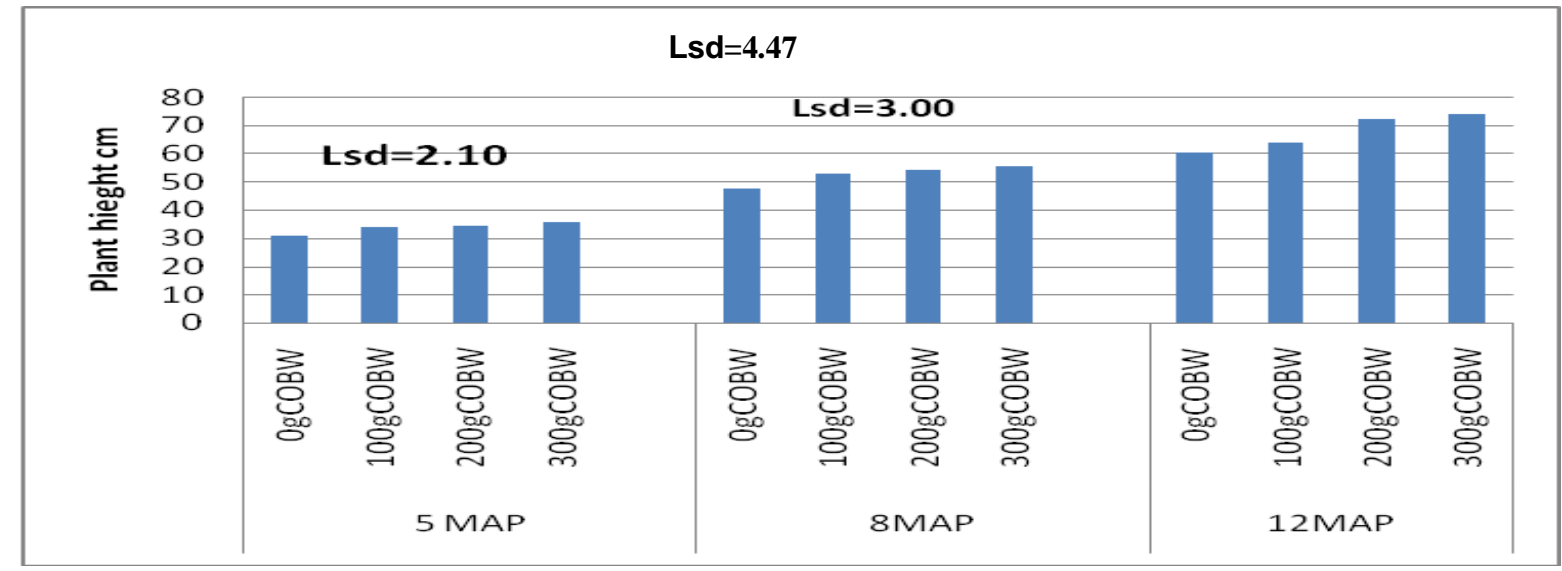

Fig 6: Main effect of composted oil palm bunch waste on plant height at 5, 8 and 12 MAP

Table 5: Effects of compost and chemical fertilizer on leaf area $\left(\mathrm{cm}^{2}\right)$ of oil palm seedlings under different irrigation intervals at 5,8 and 12 months after planting (i.e. 3, 6 and 10 months after treatment application)

\begin{tabular}{|c|c|c|c|c|c|c|c|c|c|c|c|c|}
\hline \multirow[b]{2}{*}{ Nutrient combinations } & \multicolumn{4}{|c|}{$\begin{array}{c}5 \text { MAP } \\
\text { Irrigation intervals (days) }\end{array}$} & \multicolumn{3}{|c|}{$\begin{array}{c}8 \mathrm{MAP} \\
\text { Irrigation intervals (days) }\end{array}$} & \multicolumn{5}{|c|}{$\begin{array}{c}12 \text { MAP } \\
\text { Irrigation intervals (days) }\end{array}$} \\
\hline & 7 & 14 & 21 & Mean & 7 & 14 & 21 & Mean & 7 & 14 & 21 & Mean \\
\hline $\mathrm{T} 1=0 \mathrm{gNPKMg}+0 \mathrm{gCOBW}$ & 4.14 & 5.72 & 4.78 & 4.88 & 12.74 & 11.74 & 10.23 & 11.57 & 14.08 & 15.29 & 12.28 & 13.88 \\
\hline $\mathrm{T} 2=0 \mathrm{gNPK}+100 \mathrm{gCOBW}$ & 7.98 & 6.87 & 6.41 & 7.08 & 15.91 & 13.07 & 13.08 & 14.02 & 17.91 & 19.92 & 14.57 & 17.46 \\
\hline $\mathrm{T} 3=0 \mathrm{gNPK}+200 \mathrm{gCOBW}$ & 5.28 & 5.79 & 6.39 & 5.82 & 15.33 & 13.81 & 11.21 & 13.45 & 19.11 & 20.75 & 18.21 & 19.35 \\
\hline $\mathrm{T} 4=0 \mathrm{gNPK}+300 \mathrm{gCOBW}$ & 6.78 & 6.26 & 7.61 & 6.88 & 15.97 & 14.70 & 14.01 & 14.89 & 24.31 & 19.65 & 14.35 & 19.43 \\
\hline $\mathrm{T} 5=28 \mathrm{gNPK}+0 \mathrm{gCOBW}$ & 6.17 & 5.15 & 5.05 & 5.45 & 13.19 & 13.42 & 9.38 & 11.99 & 14.83 & 16.69 & 10.56 & 14.05 \\
\hline $\mathrm{T} 6=28 \mathrm{gNPKMg}+100 \mathrm{gCOBW}$ & 5.34 & 5.47 & 5.93 & 5.58 & 14.30 & 13.05 & 11.21 & 12.85 & 16.96 & 18.59 & 14.57 & 16.71 \\
\hline $\mathrm{T} 7=28 \mathrm{gNPKMg}+200 \mathrm{gCOBW}$ & 6.39 & 7.08 & 6.15 & 6.54 & 16.65 & 16.17 & 12.17 & 14.99 & 16.99 & 20.68 & 17.00 & 18.22 \\
\hline $\mathrm{T} 8=28 \mathrm{gNPKMg}+300 \mathrm{gCOBW}$ & 7.12 & 6.70 & 5.95 & 6.59 & 25.06 & 15.99 & 11.98 & 17.67 & 21.02 & 21.64 & 15.82 & 19.49 \\
\hline $\mathrm{T} 9=42 \mathrm{gNPKMg}+0 \mathrm{gCOBW}$ & 5.84 & 5.24 & 5.62 & 5.56 & 13.91 & 12.36 & 8.88 & 11.71 & 16.11 & 15.82 & 11.25 & 14.39 \\
\hline $\mathrm{T} 10=42 \mathrm{gNPKMg}+100 \mathrm{gCOBW}$ & 6.08 & 6.74 & 5.08 & 5.96 & 16.52 & 16.07 & 9.31 & 13.96 & 26.07 & 17.46 & 15.11 & 19.54 \\
\hline $\mathrm{T} 11=42 \mathrm{gNPKMg}+200 \mathrm{gCOBW}$ & 4.45 & 6.21 & 5.83 & 5.50 & 12.47 & 18.62 & 10.79 & 13.96 & 22.50 & 23.12 & 13.03 & 19.55 \\
\hline $\mathrm{T} 12=42 \mathrm{gNPKMg}+300 \mathrm{gCOBW}$ & 6.40 & 8.05 & 6.47 & 6.97 & 14.04 & 17.37 & 10.25 & 13.88 & 20.91 & 19.92 & 14.57 & 23.10 \\
\hline Mean & 6.01 & 6.23 & 5.87 & & 15.51 & 14.70 & 11.04 & & 19.22 & 20.21 & 14.37 & \\
\hline F-LSD $0.05 \mathrm{~A}$ & \multicolumn{4}{|c|}{ NS } & \multicolumn{3}{|c|}{1.46} & \multicolumn{5}{|c|}{1.87} \\
\hline F-LSD 0.05 B & \multicolumn{4}{|c|}{ NS } & \multicolumn{3}{|c|}{ NS } & \multicolumn{5}{|c|}{ NS } \\
\hline F-LSD $0.05 \mathrm{C}$ & \multicolumn{4}{|c|}{0.63} & \multicolumn{3}{|c|}{1.69} & \multicolumn{5}{|c|}{2.16} \\
\hline F-LSD $0.05 \mathrm{~A} \times \mathrm{B} \times \mathrm{C}$ & \multicolumn{3}{|c|}{ NS } & & \multicolumn{3}{|c|}{ NS } & \multicolumn{5}{|c|}{ NS } \\
\hline
\end{tabular}


Ovie, S., Nnaji, G. U., Oviasogie, P.O., Osayande P.E. and Irhemu, P.

Table 6: Effects of compost and chemical fertilizer on leaf number of oil palm seedlings under different irrigation intervals at 5, 8 and 12 months after planting (i.e. 3, 6 and 12 months after treatment application)

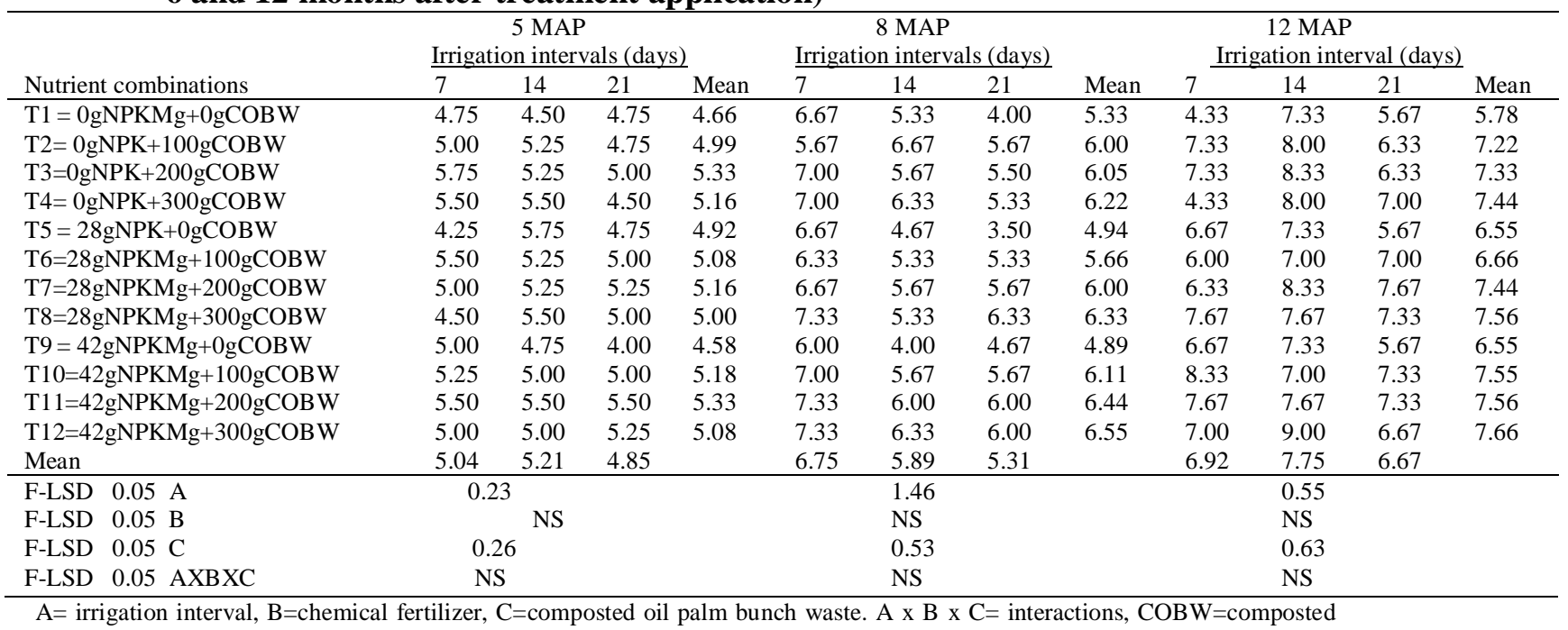

oil palm bunch waste

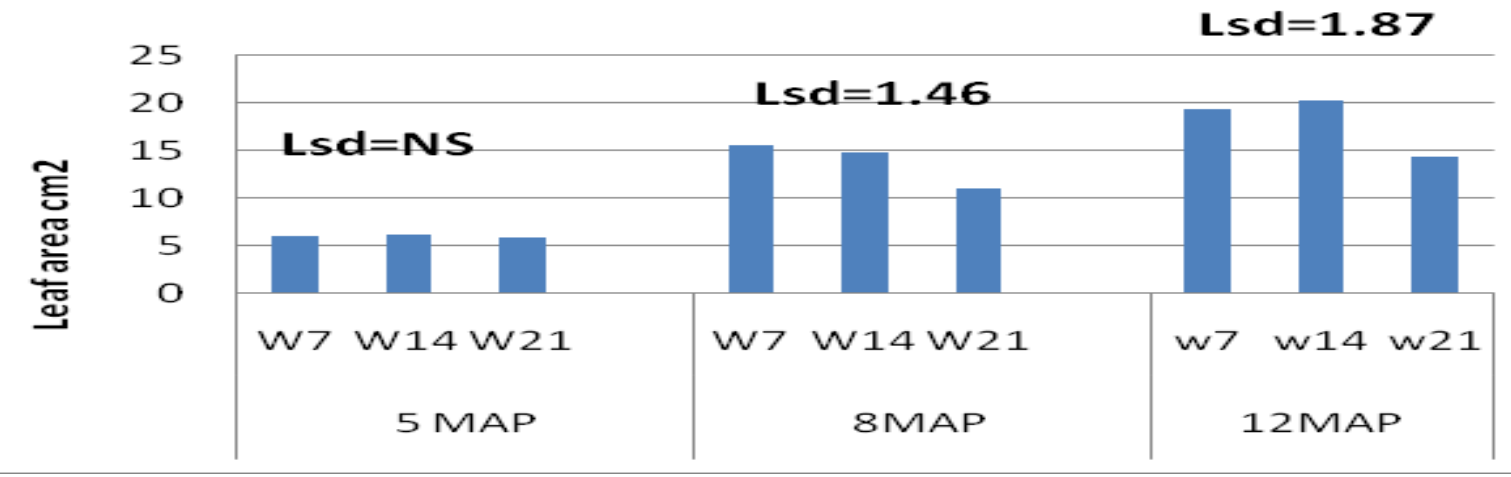

Fig 7: Main effect of irrigation intervals on leaf area at 5, 8 and 12 MAP

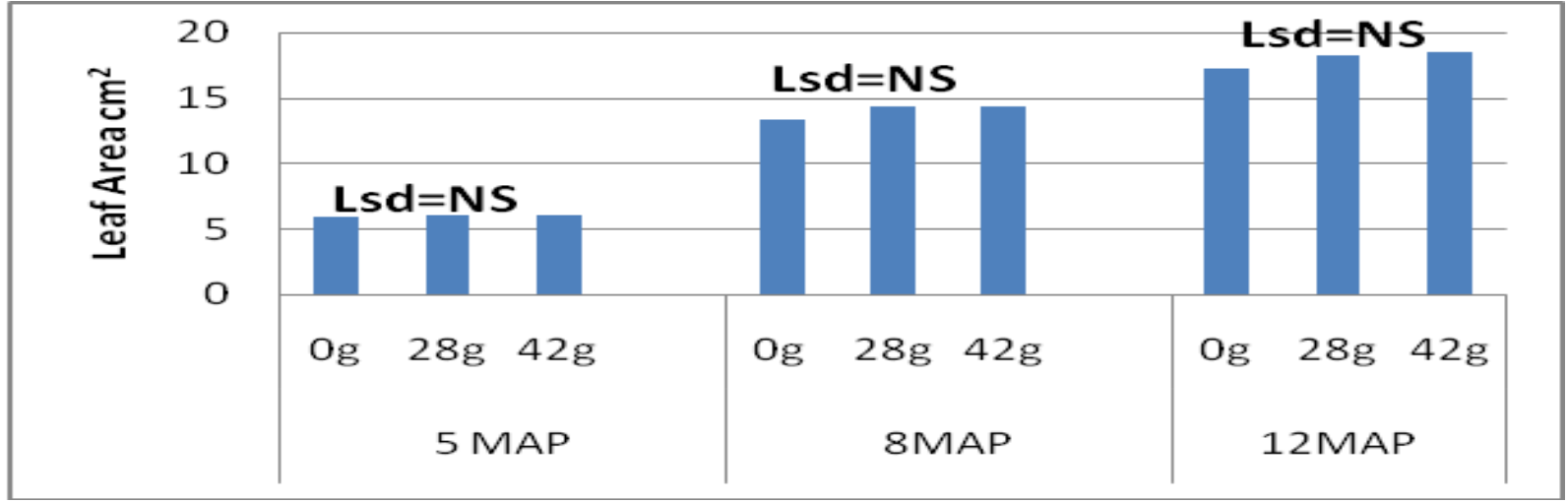

Fig 8: Main effect chemical fertilizers on leaf area at 5, 8 and 12 MAP 
Effects Of Composted Oil Palm Bunch Wastes And Chemical Fertilizer On Growth Of Oil Palm Seedling

At 8MAP, soil amended with $28 \mathrm{gNPKMg}+300 \mathrm{gcompost}$ and $28 \mathrm{gNPKMg}+200 \mathrm{gcompost}$ gave the highest value followed by those fertilized with $0 \mathrm{gNPKMg}+300 \mathrm{gcompost}$ and $0 \mathrm{gNPKMg}+100 \mathrm{~g}$ compost. Lowest values were obtained by fertilizing with $0 \mathrm{gNPKMg}+0 \mathrm{~g}$ compost, $28 \mathrm{gNPKMg}+0 \mathrm{gcompost}$ and $42 \mathrm{gNPKMg}+0 \mathrm{gcompost}$. The interaction effect between irrigation intervals, chemical fertilizer and compost was not significant $(\mathrm{P}>0.05)$ (Table 5).At 12MAP, application of 42gNPKMg+300gcompost and $42 \mathrm{gNPKMg}+200 \mathrm{gcompost}$ gave the highest values followed by those fertilized with 42gNPKMg+200gcompost,

$28 \mathrm{gNPKMg}+200 \mathrm{gcompost}$ and $0 \mathrm{gNPKMg}+300 \mathrm{gcompost}$. Lowest values were obtained by fertilizing with 0gNPKMg+0gcompost, 28gNPKMg+0gcompost and $42 \mathrm{gNPKMg}+0 \mathrm{gcompost}$. The interaction effect between compost, irrigation intervals and chemical fertilizer was not significant at 12 MAP (Table 5).

Leaf number: Figure 10 showed the main effects of irrigation intervals on leaf number of oil palm seedlings at 5, 8 and 12 MAP. At 5MAP, the highest $(\mathrm{P}<0.05)$ value was obtained at irrigation intervals of 14 days. On the contrary, the highest $(\mathrm{P}<0.05)$ value was obtained under 7days irrigation intervals at 8 MAP. At the 12MAP, highest $(\mathrm{P}<0.05)$ value was obtained under 14 days irrigation intervals (Fig. 10). Figure 11 showed that the main effect of chemical fertilizer was not statistically significant on leaf number of oil palm seedlings at 5, 8 and 12 MAP. Figure 12 showed main effects of composted EFB on leaf number of oil palm seedlings at 5, 8 and 12 MAP. At 5MAP, the highest $(\mathrm{P}<0.05)$ value was obtained at $200 \mathrm{gEFB}$ relative to the control. At 8MAP, highest value was observed at 300 gcompost. Similar trend was observed at 12MAP (Fig. 12). Table 6 showed the effects of compost and chemical fertilizer on leaf number of oil palm seedlings under different irrigation intervals at 5 , 8 and 12 months after planting. At 5MAP, highest leaf number was observed at 14 day irrigation intervals. At $8 \mathrm{MAP}$, 7day irrigation intervals recorded the highest value, while at 12MAP; 14 day irrigation intervals gave the highest leaf number value relative to 7 and 21 days irrigation intervals respectively. However, at 5MAP, fertilizing oil palm seedling with $42 \mathrm{gNPKMg}+300 \mathrm{gcompost}$ and $0 \mathrm{gNPKMg}+$ 200 gcompost gave the highest values followed by those fertilized with $42 \mathrm{gNPKMg}+$ 100 gcompost and $28 \mathrm{gNPKMg}+200$ gcompost and $0 \mathrm{gNPKMg}+300 \mathrm{~g}$ compost, respectively. Lowest values were obtained by fertilizing with $0 \mathrm{gNPKMg}+$ 0gcompost, $28 \mathrm{gNPKMg}+$ 0 gcompost and $42 \mathrm{gNPKMg}+0 \mathrm{gcompost}$. The interaction effect between compost, irrigation intervals and chemical fertilizer were not significant $(\mathrm{P}>0.05)$. At $8 \mathrm{MAP}$ (Table 6), soil amended with $42 \mathrm{gNPKMg}+300 \mathrm{gcompost}$ and $42 \mathrm{gNPKMg}+200 \mathrm{gcompost}$ gave the highest values followed by those fertilized with $28 \mathrm{gNPKMg}+300 \mathrm{gcompost}$ and $0 \mathrm{gNPKMg}+$ 300 gcompost. Lowest values were obtained by fertilizing with $0 \mathrm{gNPKMg}+0$ gcompost, $28 \mathrm{gNPKMg}+0 \mathrm{gcompost}$ and $42 \mathrm{gNPKMg}+$ Ogcompost, respectively. The interaction effect between compost, irrigation intervals and chemical fertilizer was not significant $(\mathrm{P}>0.05)$ at 12 MAP.

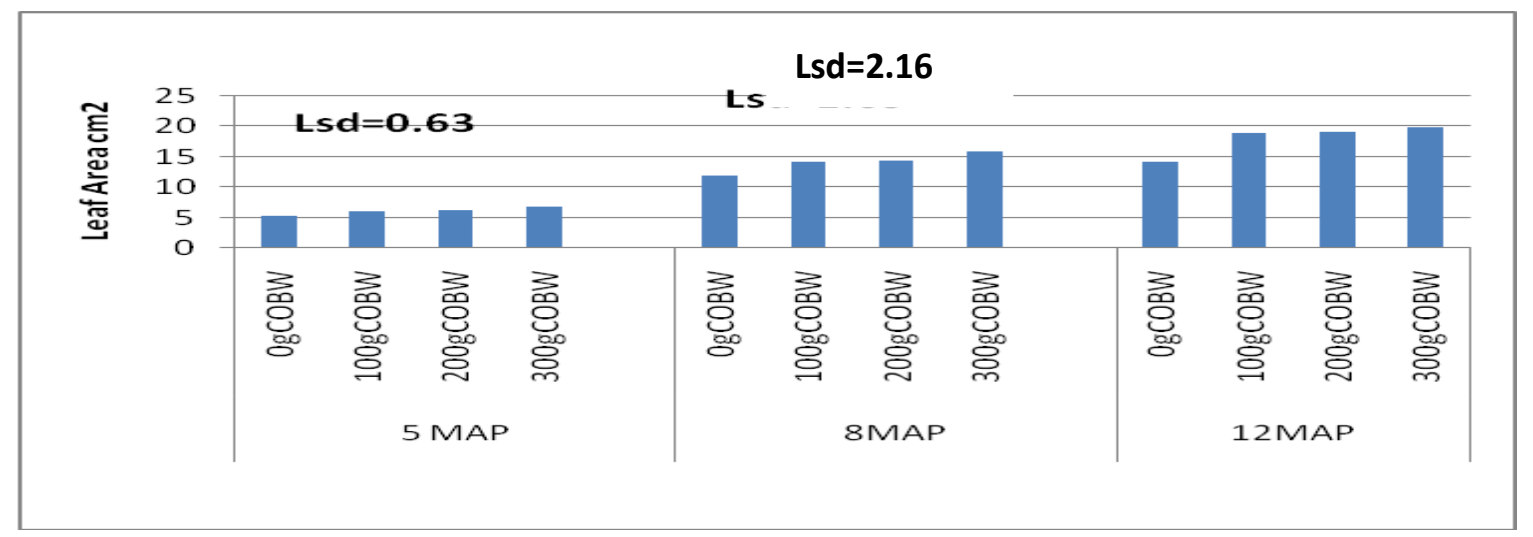

Fig 9: Main effects of composted oil palm bunch waste on leaf area at 5, 8 and 12 MAP 


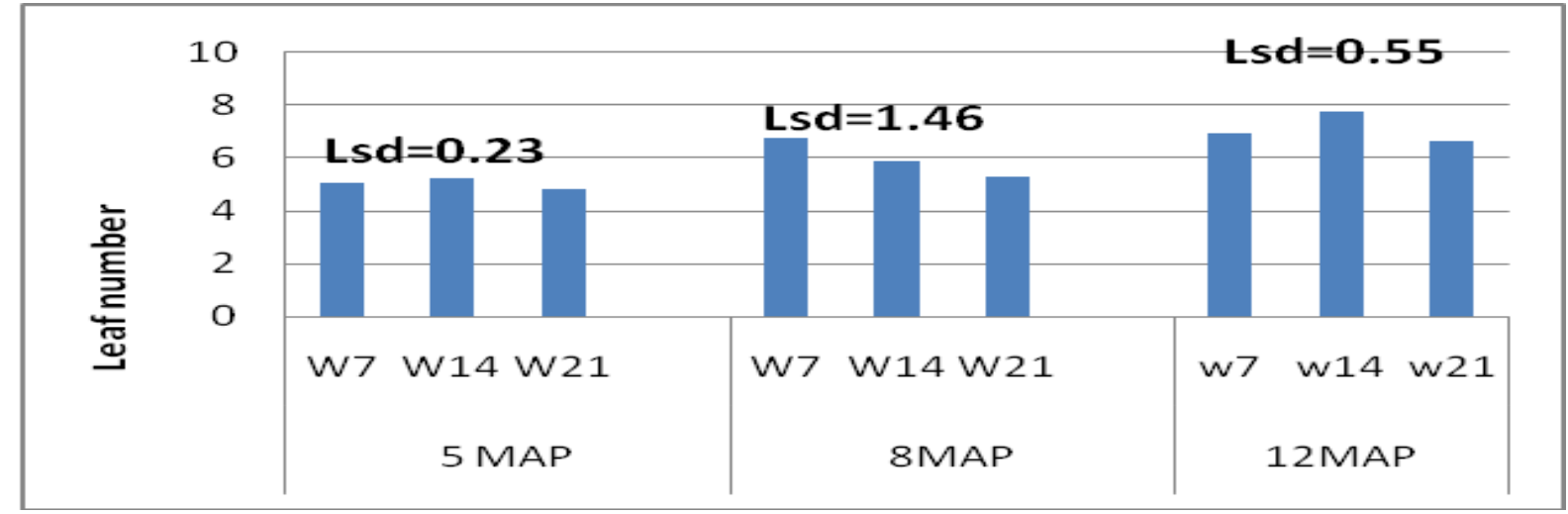

Fig 10: Main effect of irrigation intervals on leaf number at 5,8 and 12 MAP

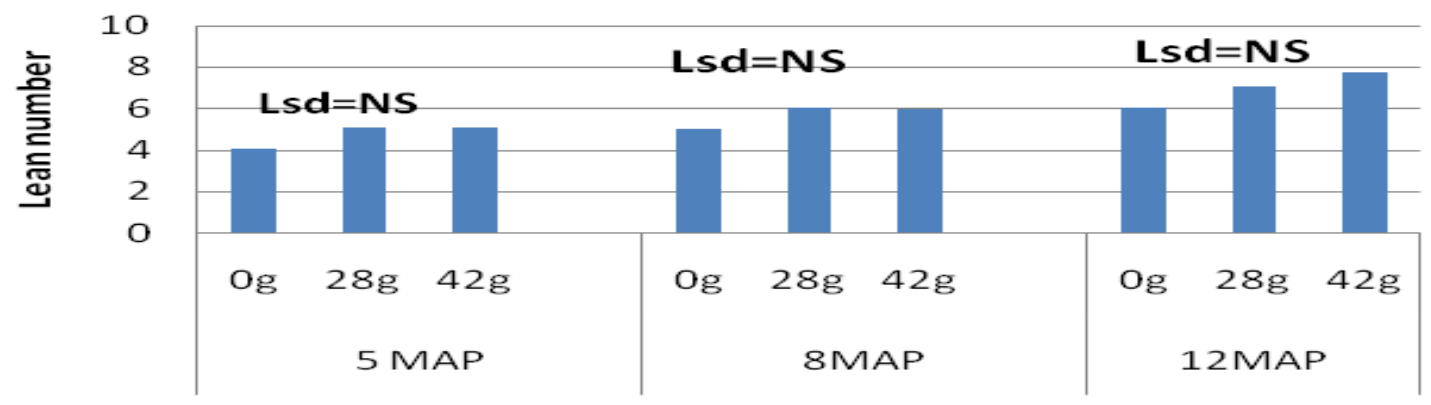

Fig 11: Main effect chemical fertilizer on leaf number at 5,8 and 12MAP

$L s d=0.63$

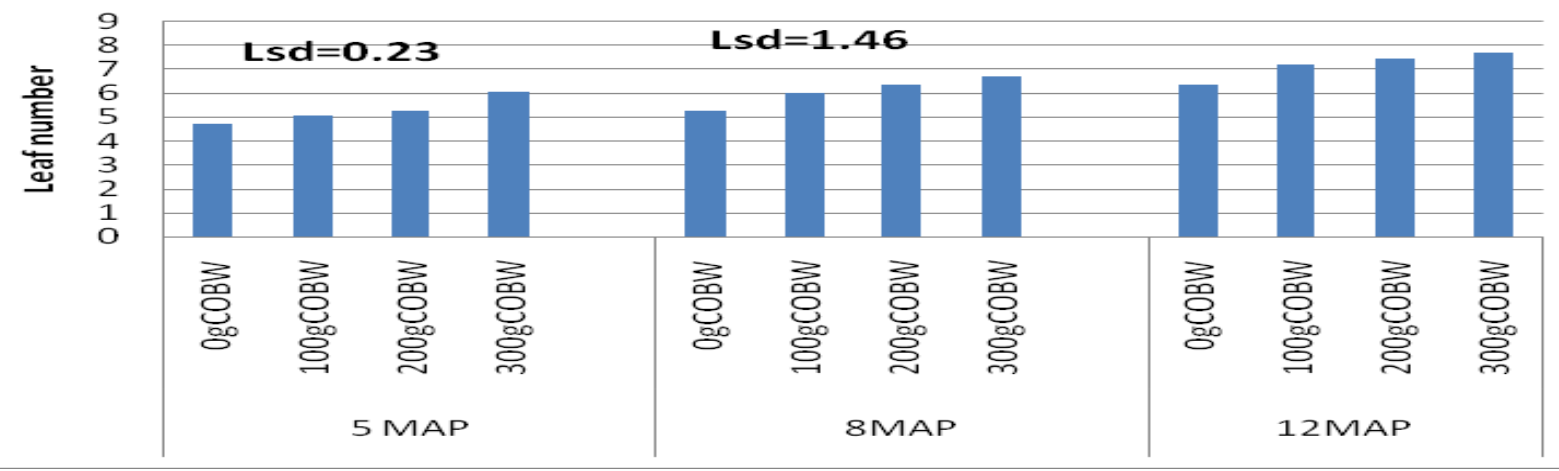

Fig 12: Main effects of composted oil palm bunch waste on leaf number at 5, 8 and 12 MAP 
At 12MAP, fertilizing with $42 \mathrm{gNPKMg}+300 \mathrm{gcompost}$ and $42 \mathrm{gNPKMg}+200 \mathrm{~g}$ compost gave the highest values followed by those fertilized with $0 \mathrm{gNPKMg}+300 \mathrm{gcompost}$ and $28 \mathrm{gNPKMg}+300 \mathrm{gcompost}$. Lowest values were obtained by fertilizing with 0gNPKMg+0gcompost, 28gNPKMg+0gcompost and $42 \mathrm{gNPKMg}+0 \mathrm{gcompost}$. The interaction effect between irrigation intervals, chemical fertilizer and compost was not significant $(\mathrm{P}>0.05)$ at 8MAP (Table 6).

\section{DISCUSSION}

The relative high sand content of soil used for the study is a reflection of a sandy parent material. The parent materials of the soils of eastern Nigeria have been noted to influence the texture of the native soils (Akamigbo and Asadu, 1983). The relatively low $\mathrm{pH}$, total $\mathrm{N}$, available $\mathrm{P}$, exchangeable $\mathrm{K}, \mathrm{Ca}, \mathrm{Mg}$ and soil organic matter content of the experimental soil confirmed the soil to be low in fertility (Ibeawuchi et al., 2006). The observed improvements in nutrient composition of the composted material relative to the raw materials may be adduced to increased organic matter mineralization and humification brought about by the action of micro-organisms. Brady and Weil (2002) reported that finished compost is generally more concentrated in nutrients than the initial combination of raw material used.

The significant improvements in growth parameters of oil palm seedlings that were mostly observed under irrigation intervals of 14 days throughout the sampling periods may be adduced to favorably soil properties. On the other hand, the poorer growth of oil palm seedlings observed under 7 days irrigation intervals may be due to applied irrigation at short intervals which led to increased soil moisture content that may lead to weakening and breakdown of aggregate resulting in rearrangement of soil particle, reorientation of soil pores and increased compaction and plant root restriction. These results were in agreement with those reported by Haynes and Swift (1990). Similarly, the shortest irrigation intervals of 7 days may have resulted to increase leaching of mineralized soil nutrient and possibly increase acidification. The extension of irrigation to 21 days showed poorest growth of oil palm seedling throughout the sampling period. This phenomenon may be attributed to the negative effects of water stress. Irrigation intervals of 21 days may have resulted in the decline in soil nutrient availability, their movement (mass flow and diffusion) and uptake by the oil palm seedlings. Guiterrez-Boen and Thomas (1999) reported that the decrease in soil water availability affects the rate of diffusion of many plant nutrients and finally their composition and concentration. The reduction of plant size and growth under water stress may be attributed to a decrease in the activity of meristemic tissues responsible for elongation (Siddique at al., 1999). Water stress conditions have been found to disrupt several physiological processes leading to reduction in growth and chemical composition of sugar beet (Bloch and Hoffman, 2005).

The observed increase in growth of oil palm seedlings as affected by sole application of NPK fertilizer relative to the control may be attributed to improved soil fertility linked with the availability of the macro elements. Inorganic fertilizer exerts strong influence on plant growth, development and yield (Stefano et al., 2004). This observation is also in agreement with the findings of Babatola (2006) who reported that increasing levels of chemical fertilizer application was observed to increase growth and yield of crops. The unamended seedlings were almost stunted in growth as they had to rely on the native soil fertility which from the result of initial chemical analysis was deficient in these nutrients

Improvements in growth of oil palm seedling were also observed with soil application of compost relative to the control. The improved growth may be attributed to increased microbial activities and mineralization of nutrients induced by composted oil palm bunch waste addition. Giwa and Ojeniyi (2004) reported that organic material improved tomato growth by increased soil organic matter, total $\mathrm{N}$, available $\mathrm{P}$ and exchangeable $\mathrm{K}, \mathrm{Ca}$ and $\mathrm{Mg}$ availability respectively. The $\mathrm{C} / \mathrm{N}$ ratio of 17.85 as observed with the composted oil palm bunch waste may have enhanced nutrients mineralization. This is line with the findings of Olayinka (2001) who observed that organic material having $\mathrm{C} / \mathrm{N}$ ratio less than 30:1 encourages faster organic matter decomposition and ease of nutrient release. The result of this study further corroborates the findings of Ahmad et al. (2008) who reported that composed poultry manure improved growth of plants due to the readily available nitrogen it supplies.

Under water stress conditions, complementary use of compost and chemical fertilizer likewise those plants amended with compost alone showed unique improvements in growth parameters compared to the use of chemical fertilizer alone and the control. These unique attributes may be in connection with the fact that the applied compost singly or in combination with the chemical fertilizer positively influenced the organic matter and other soil physical, chemical and biological properties (El-bady and 
Asaad, 1983). Similarly, Woomer et al. (1994) and Hossner and Juo (1999) reported that compost material improves soil structure, increases water holding capacity of soils, increases cation exchange capacity (CEC) and increases the capacity of low activity clays to buffer changes in $\mathrm{pH}$. In addition, Uyobisere and Elomo (2002) noted the superior effect of integrated nutrient use over sole use of inorganic or organic source in terms of improved soil fertility and crop performance. The observed growth improvements of oil palm seedlings by combined application of organic and inorganic fertilizer may also be as a result of favorable soil $\mathrm{pH}$ and better nutrient supply (Lopez et al., 1996; Yamagata and Otami, 1996).

\section{CONCLUSION}

The study showed that under moisture stress condition the growth of oil palm seedlings were enhanced by combined use of composted oil palm bunch refuse and chemical fertilizer, compared to sole use of compost, chemical fertilizer and the control. Results revealed that every 14 days interval of irrigation significantly improved the growth parameters compared to 7 and 21 days intervals of irrigation throughout the sampling period. The application of $42 \mathrm{gNPKMg}$ fertilizer showed highest $(\mathrm{P}>0.05)$ effects on all growth parameters relative to other NPKMg rates. Result also indicated that sole application of 300 gcompost/seedlings gave highest $(\mathrm{P}<0.05)$ effect on all growth parameters of oil palm seedlings throughout the sampling period compared to other compost rates. The interaction between compost, irrigation intervals and chemical fertilizer was not significant at all samplings times. On the Average, combined application of $42 \mathrm{gNPKMg}$ compound fertilizer and 300 gcompost/seedlings was most effective in improving the growth of oil palm seedlings under moisture stress imposed at 14 day intervals of irrigation and is therefore recommended.

\section{REFERENCES}

Ahmad, R., M. Arshad, Z. A., Zahir, M., and Asghar, H. N. (2008). Integrating nenriched compost with biologically active substances for improving growth and yield of cereals. Pakistan Journal of Botany. 1:283-293.

Aisueni, N.O. Omoti U., Ekhator F., and Oviasogie, P.O. (2000). Effect of compost on soils supporting nursery seedling production of oil palm. Nigeria Journal of Crop Research.4(2):15-20

Akamigbo, F. O. R. and Asadu, C. L. A. (1983). The accuracy of field textures in a humid tropical environment. Soil
Survey and Land Evaluation.4(3):6370.

Akamigbo, F.O.R., and Igwe C.A. (1990). Morphology, genesis and taxonomy of three soil series in eastern Nigeria. Samaru Journal of Agricultural Research.7:33-48.

Aoyama, M., Angers, D.A. and Dayegamiye, A.N. (1999). Particulate and mineral associated organic matter in waterstable aggregates as affected by mineral fertilizer and manure applications. Canadian Journal of Soil Science.79:295-302.

Asadu, C.L.A. (2002). Fluctuations in the characteristics of an important short tropical season "August Break" In eastern Nigeria. Discovery and Innovation. 14:92-101.

Ayeni, L.S. (2008). Intergrated application of cocoa pod ash and NPK fertilizer on soil chemical properties and yield of tomato. American Journal of Sustain Agric. 2(3):333-3337.

Ayoola, O.T. and Agboola, A.A. (2002). Influence of cassava planting pattern and organic and inorganic fertilizer sources on the growth and yield of maize in cassava/maize/melon intercrop in South-West Nigeria. Moor Journal Agric. Res. 3(2):161-168.

Babalola and Obi, M.E. (1981) Physical properties of acid sand in relation to land use. In:Udo,E.J. and Sobulo, R.A. (ed) Acid sands of Southern Nigeria. Soil Science Society of Nigeria Special Publication Monography.1: 27-55.

Babatola, I. A. (2006). Effects of NPK 15:15:15 fertilizer on the performance and storage life of okra (Abelmuschus esculentus). Proceedings of the Horticultural Society of Nigerian Conference. Pp. 125-128.

Blair, N.R., D. Fulkner, Till, A.R. and Poulton, P.R (2005). Long-term management impacts on soil carbon, nitrogen and physical fertility. Soil and Tillage. Research. 91:30-38.

Bloch, D. and Hoffman, C. (2005). Seasonal development of genotypic differences in sugar beet and their interaction with water supply. Journal of Agronomy and crop science. 191 (4): 263 - 272.

Boutraa, A and F. E, Sanders (2011). Influence of water stress in grain yield and vegetable growth of two cultivars of bean (Phasoeolus/Vulgaris L.). Journal of Agronomy and Crop Science. 186:222-237. 
Bouyoucos, G.J. (1962). Hydrometer methods improved for making particle size analysis of soils. Proceedings of Soil Science Society of America.26:464-465

Brady N. C. and R. R. Weil (2002). The Nature and Properties of Soils. Thirteenth Edition. Pearson Education. Page 976.

Bray, R.H., and Kurtz (1945). Determination of total organic and available forms of phosphorus in soils. Soil Science.59:3945

Bremner, J. M. (1996). Nitrogen-Total. In: D.L. Sparks (Editor) Methods of Soil Analysis Part 3-Chemical Methods, Soil Science Society of America. Book Series 5, Madison, Wisconsin, USA. Pp $1085-1122$.

Catton, C. (1983): The Oil Palm. Longmans London $3^{\text {rd }}$ edition.

Cheng, F.J.,Yang, D.Q. and Wu, Q.S. (1998). Physiological effects of humic acid on drought resistance of wheat. Chinese Journal of Applied Ecology. 6:363 367.

Corley, R.H.V. (2009). How much palm oil do we need? Environ. Science Polic. 12:134-139.Corley, R.H.V. and Tinker, P.B. (2009). The Oil Palm $4^{\text {th }}$ Edition. Uk: Blackwell Pub.

El-bady, D.D. and Asaad F. (1983). Improving water retention of sandy soils through the addition of farmyard manure. Egyptian Journal of Soil Science.23:9193.

Giwa, D. D. and Ojeniyi, S. O. (2004). Effect of integrated application of pig manure and NPK on soil nutrient content and yield of tomato. Proceedings $29^{\text {th }}$ Annual Conference of Soil Science Society of Nigeria, University of Agricultural, Abeokuta (UNAAB). Pp.164-169.

Grant, W. T. (1982). Exchangeable cations. In : A Page et al (Eds). Methods of Soil Analysis. Part 2, 2nd (Ed). Agron. Mone ASA and SSSA, Madison W. I.

Guitierrez-Boem, F. H. and Thomas, G. W. (1999).Phosphorus nutrition and water deficits in field grown soya beans. American Journal of Soil Science. 207: 87-96.

Hang, R.T. (1993). The practical handbook of compost engineering, Lewis Publishers, ISBN 0-87371-373-7, Boca Raton (Florida)

Harden, J.J., Williams C and Watson I (1965). Leaf area and yield of the oil palm in Malaysia. Experimental Agric.5:25-32.

Hartley, C.W.S. (1988). The oil palm 3rd Edition Longman England, 761p.
Hossner, L.R, and Juo, A.S.R. (1999). Soil nutrient management for sustained food crop production in upland farming systems in the tropics. Juo Soil and Crop Sciences Department Collage Station Tennessee 77843, USA. Retrieved from http/www.agnet.org.

Ibeawuchi, I. I., Onweremadu, E. U. and Oti, N. N. (2006). Effects of poultry manure on green (Amaranthus cruentus) and degraded ultisol of Owerri, Southeastern Nigeria. Journal of Animal and Veterinary Advances. 5(1):53-56.

Idoga, S., Adogoye, M.S. and Ogbonnaya, J.M. (1998). Changing climatic conditions and agricultural production implications in Benue State, Nigeria. Proceedings of the 23rd Annual Conference of Soil Science Society of Nigeria from April 12-16: Pp 59-62.

Isenmila, A.E. (1991). Effect of soil moisture stress on subsequent growth, water relations and dry matter production of oil palm seedling. Nigeria Journal of Palms and Oil Seeds. 12: 21-26.

Kundu, S.R., Bhattacharyya, V., Parkash, B.N., Ghosh and Gupta, H.S. (2006). Carbon sequestration and relationship between carbon addition and storage under rainfed soybean-wheat rotation in a sandy loam soil of the Indian Himalayas. Soil and Tillage Research.92:87-95.

Lopez, A. J., Do R., Stanford, N. P. and Ferraz, I. B. (1996). Effect of application of urban compost, mineral N. and mineralizing agent of the $\mathrm{N}$ fixation on yield of cowpea. Rev. Bras. Cienic. Solo. 20(1):55-62.

Mannions, A.M. (1998). Future trends in agriculture: The role of biotechnology. Outlook Agric.27:219-224.

Mclean, L. O. (1965). Aluminuim in L.A Black (ed) Methods of Soil Analysis.Part 2 American Society of Agronomy. Madison. 1:976-985.

Nabizade, M., Saki Nejad, T. and Mojadem, M. (2011). Effect of irrigation on the yield of mangbean cultivers. American Journal of Soil Science.7(7):86-90.

Nelson, D. W. and Sommers, L. E. (1982). Total carbon, organic carbon and organic matter. In: D.L. Sparks (Editor). Methods of Soil Analysis Part 3Chemical Methods, American Society of Soil Science, Book Series 5, Madison, Wisconsin, USA. Pp 9611010 . 
Okusami, R.A., Rust, R.H. and Alao, A.O. (1997). Red soils of different origins from southwest Nigeria characteristics, classification and management considerations. Canadian Journal Soil Science.77:295-307.

Olayinka A (2001). Environmentally-friendly means of managing poultry litter. Invited paper presented at the Workshop "Improving Poultry Feed Supply and Litter Management" organized by Worlds Poultry Science Association, Nigeria Branch in Partnership with the Department of Animal Science, Obafemi Awolowo University, Ile-Ife, Nigeria, February $15,2001$.

Olayinka A (2009). Soil microorganisms, wastes and national food security. Inaugural Series 222. Obafemi Awolowo University, Ile-Ife, Nigeria.

Onwubuya, I.I. (1982) Preliminary trials on the fertilizer requirements of the polybag oil palm seedlings. Journal Nigeria Institute Oil palm Research. 6(22):205 -215 .

Siddique, M. R., Hamid, B. and Islam, M. S. (1999). Drought stress effect on photosynthetic rate and leaf gas exchange of wheat. Botanical Bull of Academic Sinica: 40 (20):141-145.

Stefano, P., Dris, R. and Rapparini, F. (2004). Influence of growing conditions and yield and quality of cherry. Journal of
Agriculture and Environment. 2:307309.

Turner, P.D. (1976).The effects of drought on oil palm yields in south-east Asia and south pacific region. Proceedings of the Malaysia Developments in Oil palm Conference Kuala Lumpurp, June 1417.Pp. 673-694.

Ugbah, M.M. (2008). Preliminary study of the response of oil palm seedlings to irrigation and fertilizer levels. Nigeria Agricultural Journal. 39(1):76-78.

Uyovbisere, E.O and Elemo, K.A. (2000). Effect of inorganic fertilizer and foliage of Azadirachta and Parkia species on the productivity of early maize. Nigerian. Journal Soil Resource.1:17-22.

Woomer, P.L, Martin, A., Albrecht, A., Recsk, D.V.S., Scharpenseel, H.W. (1994). The importance and management of soil organic matter in the tropics. In: The biological management of tropical soil fertility P.L. Woomer and M.J. Swift (Eds.). Chichester, UK. WilleySayce. Pp. 47-80.

Yamagata, M. A. N. and Otami (1966). Nitrogen uptake response of crops to organic nitrogen. Japan Journal Soil Science Plant Nutrition: 67(4):345-353.

Yusuf, B. (2007). Palm importation through sustainable plantations. European Journal Lipid Science Technology. 109:289-295. 